\title{
In-situ Beschreibung des Wurzelsystems von Hopfen und Mais über Freilegung am Bodenprofil
}

\author{
In-situ root system characterization of hop and \\ maize via soil profile excavation
}

\author{
Monika Sobotik ${ }^{1}$, Tobias Graf ${ }^{2}$, Margarita Himmelbauer ${ }^{3}$, Gernot Bodner $^{4 *}$, Andreas Bohner ${ }^{5}$, \\ Willibald Loiskandl ${ }^{3}$
}

\footnotetext{
${ }^{1}$ Pflanzensoziologisches Institut, 4822 Bad Goisern, Pichlern 9, Österreich

${ }^{2}$ Lehrstuhl für Pflanzenernährung, Wissenschaftszentrum Weihenstephan, Technische Universität München, Emil-Ramann-Straße 2, 85354 Freising, Deutschland

${ }^{3}$ Institut für Hydraulik und landeskulturelle Wasserwirtschaft, Department für Wasser-Atmosphäre-Umwelt, Universität für Bodenkultur Wien (BOKU), Muthgasse 18, 1190 Wien, Österreich

${ }^{4}$ Abteilung Pflanzenbau, Department für Nutzpflanzenwissenschaften, Universität für Bodenkultur Wien (BOKU), Konrad-Lorenz-Straße 24, 3430 Tulln, Österreich

${ }^{5}$ Institut für Pflanzenbau und Kulturlandschaft, Höhere Bundeslehr- und Forschungsanstalt Raumberg-Gumpenstein, Raumberg 38, 8952

Irdning, Österreich

* Korrespondierender Autor: gernot.bodner@boku.ac.at
}

Einreichung: 30. Juli 2018, überarbeitete Einreichung: 14. August 2018, Annahme: 14. August 2018

\section{Zusammenfassung}

Im Rahmen der Tagung „Wurzel und Rhizosphäre: Ökophysiologie, Humushaushalt und Bodenmanagement” im September 2015 wurden Wurzelfreilegungen von Hopfen und Mais in Wolnzach (Bayern) durchgeführt. Das Hauptziel der Untersuchungen war, die Möglichkeiten und den Wert von Wurzelfreilegungen im Feld zu demonstrieren. Für die Hopfenfreilegungen konnten die sichtbaren oberirdischen Unterschiede auch durch die Bewurzelungsweise erklärt werden. Der Einfluss der Bewässerung auf das Wurzelwachstum wurde ebenfalls untersucht. Die schwächer entwickelten Hopfenpflanzen wiesen eine Wurzeltiefe von ca. $130 \mathrm{~cm}$ auf, während die Wurzeln der gut entwickelten Pflanzen bis in eine Tiefe von $370 \mathrm{~cm}$ verfolgt werden konnten. Weiters wurden Maispflanzen bei konservierender Bodenbearbeitung mit Direktsaat untersucht. Im Gegensatz zu anderen Standorten konnten mehr Sprosswurzeln und lange Seitenwurzeln bis in eine Bodentiefe von $140 \mathrm{~cm}$ gefunden werden.

Schlagworte: Wurzelsystem, Hopfen, Mais, Bewässerung, Wurzelanatomie

\begin{abstract}
Summary
As part of the workshop "Root and rhizosphere: Ecophysiology, humus condition and soil management" which was conducted in September 2015, root excavations of hops and maize were performed in Wolnzach (Bavaria). The main intention was to demonstrate the possibilities and the value of field root excavation on real world cases. A question if the above-ground difference in hops growth due to irrigation is reflected in the root development was verified. The poorly developed hop plants had shallow rooting depth of about $130 \mathrm{~cm}$, while the plants with apparently higher biomass developed abundant root systems down to $370 \mathrm{~cm}$ depth. The development of maize plants in conservation agriculture farming using direct seeding was also investigated. In contrast to other sites, more shoot roots and long side roots were found to grow deeply reaching $140 \mathrm{~cm}$ soil depth.
\end{abstract}

Keywords: Root system, hops, maize, irrigation, root anatomy 


\section{Einleitung}

Die Ausprägung des Wurzelsystems von Nutzpflanzen beeinflusst deren Wachstum und Ertragsleistung unter variablen Umweltbedingungen und wird durch zahlreiche pflanzenbauliche Managementmaßnahmen beeinflusst (z. B. Lynch, 1995; Bodner et al., 2015). Im Rahmen der gemeinsamen Tagung „Wurzel und Rhizosphäre: Ökophysiologie, Humushaushalt und Bodenmanagement" vom 14./15. September 2015 in Pfaffenhofen an der Ilm der Österreichischen Gesellschaft für Wurzelforschung (ASRR), der Fördergesellschaft für Agrarwissenschaften und der Gesellschaft für Konservierende Bodenbearbeitung (GKB) erfolgten an zwei Standorten Freilegungen des Wurzelraums von Hopfen (Humulus lupulus L. cv. Perle) und Mais (Zea mays L. cv. LG 30222).

Zweck der zwei Hopfenfreilegungen war, die sichtbaren oberirdischen Unterschiede auch durch die Bewurzelungsweise erklären zu können. Die dort schon seit vielen Jahren deutlich schwächere oberirdische Entwicklung hoffte man durch eine $30 \mathrm{~cm}$ tiefe, unterirdische Tropfbewässerung verbessern zu können (Abbildung 2). Der Einfluss der Bewässerung auf das Wurzelwachstum sollte ebenfalls untersucht werden. Die ausgedehnte Bewurzelung der Hopfenpflanzen benötigt tiefgründige, gut durchwurzelbare Böden, die in der Hallertau, dem größten zusammenhängenden Hopfenanbaugebiet der Welt, vorhanden sind (Rid, 1966; Kohlmann und Kastner, 1975). Bevorzugt werden gut bearbeitbare, mittlere und leichtere Böden. Schwere Böden können zu Staunässe neigen und somit zu Wachstumsstörungen führen.

Für die Maisausgrabungen war entscheidend, dass in der Vergangenheit vom Pflanzensoziologischen Institut schon zahlreiche Freilegungen von Maispflanzen auf Flächen mit Pflugbewirtschaftung und mit konservierender Bodenbearbeitung mit Direktsaat durchgeführt worden waren. Eine Referenz in der Hallertau war eine willkommene Ergänzung, und auch die Gesellschaft für konservierende Bodenbearbeitung wollte diesen Standort, der nach ihren Vorstellungen bearbeitet wird, näher betrachten.

\section{Material und Methoden}

\subsection{Standort und Bodendaten}

Wolnzach (Landkreis Pfaffenhofen an der Ilm, Bayern) liegt in der Kulturlandschaft Hallertau, dem größten zusammenhängenden Hopfenanbaugebiet der Welt. Das
Klima in der Hallertau ist gemäßigt, aber kalt. Die Klassifikation des Klimas nach Köppen und Geiger ist Dfb (Feuchtes kontinentales Klima). In Wolnzach herrscht im Jahresdurchschnitt eine Temperatur von $8,1^{\circ} \mathrm{C}$. Über das Jahr verteilt gibt es im Schnitt $780 \mathrm{~mm}$ Niederschlag (CLIMATE-DATA.ORG, 2015). Die vorherrschenden Bodentypen in der Region sind Braunerden und Parabraunerden. Für die beiden Standorte Hopfen (2 Profilgruben) und Mais wurden bodenchemische und bodenphysikalische Daten analysiert. Vor Ort wurde eine Bodenansprache durchgeführt und Bodenproben entnommen. Die Proben wurden am Institut für Hydraulik und landeskulturelle Wasserwirtschaft der Universität für Bodenkultur Wien (BOKU) analysiert. Nach den üblichen Standardmethoden wurden die Korngrößenverteilungen (ÖNORM L1061-1, ÖNORM L1061-2), der Wassergehalt (gravimetrisch), der pH-Wert $\left(\mathrm{CaCl}_{2}\right)$, die elektrische Leitfähigkeit, der Gesamtstickstoff (Carlo Erba CNS Analyzer), der Phosphor als $\mathrm{P}_{2} \mathrm{O}_{5}$ (ÖNORM L 1087) und der Bodenkohlenstoff (Gesamtkohlenstoff $\mathrm{C}_{\text {tot }}$ CNS Analyzer; anorganischer Kohlenstoff $\mathrm{C}_{\text {anorg }}$, Calcimeter nach Scheibler; organischer Kohlenstoff $\mathrm{C}_{\text {org }}=\mathrm{C}_{\text {tot }}-\mathrm{C}_{\text {anorg }}$ ) bestimmt.

\subsection{Wurzelfreilegung}

Für die Wurzelfreilegung wurde zuerst mit einem Bagger eine Profilgrube angelegt und dann wurden in Handarbeit schrittweise die Wurzeln freigelegt, unter Verwendung von Schaufeln und Spatengabeln (Abbildung 1). Die einzelnen Wurzeln wurden mit Nägeln möglichst in ihrer natürlichen Position fixiert. Die freigelegten Wurzeln wurden maßstabgetreu abgezeichnet und auch fotografisch festgehalten.

Für den Hopfen wurden zwei Gruben mit einer unterschiedlichen Tiefe, entsprechend der grob ersichtlichen Wurzeltiefe der beiden Hopfenpflanzen, ausgehoben. Die Tiefe der Profilgruben auf der nicht bewässerten Fläche lag bei $450 \mathrm{~cm}$, auf der bewässerten Fläche bei $250 \mathrm{~cm}$. Die Seitenbreiten betrugen bei beiden Gruben etwa $200 \mathrm{~cm}$.

Für die Freilegung der Wurzeln der Maispflanzen dreier benachbarter Reihen war die ausgebaggerte Grube so angelegt, dass der seitliche Abstand verschieden war. So war es möglich, sowohl rasch die Wurzeltiefen als auch ungestört die seitliche Ausbreitung von Seitenwurzeln bis in die Tiefe zu verfolgen.

Zur Dokumentation wurden wurzelspezifische Daten (mittels des Bildbearbeitungsprogrammes WinRHIZO; 


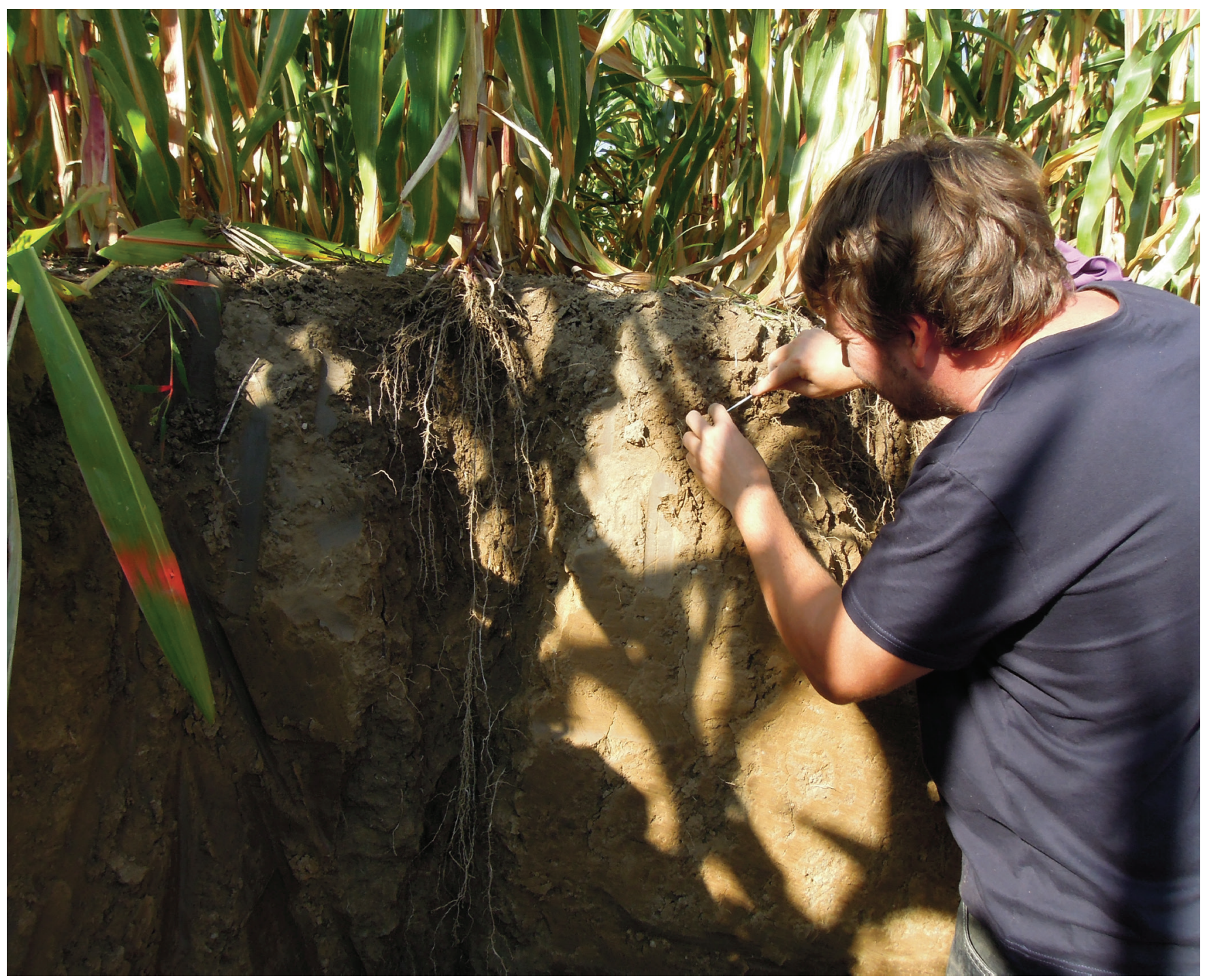

Abbildung 1. Freilegung von Maiswurzeln in Wolnzach, Hallertau

Figure 1. Excavation of maize roots in Wolnzach, Hallertau

Himmelbauer et al., 2012) erfasst und anatomische Schnitte angefertigt. Für die anatomischen Untersuchungen dienten spezifisch ausgesuchte Wurzelstücke von jungen und älteren Wurzeln. Die Schnitte wurden von Hand mit der Rasierklinge hergestellt und größtenteils ungefärbt untersucht. Einige wurden zum Nachweis von Stärke mit einer 2\%igen Jod-Kaliumjodid-Lösung (Braune et al., 1999) behandelt. Fotografiert wurden die Schnitte mit einem AXIOMAT Photomikroskop von Zeiss. Für Mais wurde eine Seitenwurzel 1. Ordnung für die Analyse mit WinRHIZO entnommen. Ausgewertet wurden die Längen, die Oberfläche und die mittleren Durchmesser der Wurzeln.

\subsection{Hopfen}

Die Bewirtschaftung (Bodenbearbeitung, Düngung und Pflanzenschutzmaßnahmen) erfolgte nach den Angaben des „Grünen Heftes“ (2015) in beiden Hopfengärten (Koordinaten: 48.572172 N, 11.649390 E), welche 25 m voneinander entfernt liegen, gleich. Die Stickstoffgaben betrugen $146 \mathrm{~kg} / \mathrm{ha}$. Bei gleichem Pflanzmaterial (Humulus lupulus cv. Perle) wurden erhebliche Ertrags- und Habitusunterschiede festgestellt. Die schlecht entwickelten Pflanzen sollten daher mit einer Tropfbewässerung unterstützt werden. Das Alter der nicht bewässerten Hopfenpflanze betrug 4 Jahre und das der bewässerten 6 Jahre. 


\subsection{Mais}

Die Wurzelgrabungen fanden an ca. 5 Monate alten Maispflanzen (Zea mays) der Sorte LG 30222 statt. Der Standort zeichnet sich durch eine pfluglose Bodenbearbeitung seitens des Landwirts in Absprache mit dem AELF in Pfaffenhofen aus (Koordinaten: 48.590968 N, 11.647338 E). Vor der Maisaussaat Anfang Mai 2015 wuchs eine Zwischenfrucht, die aus 20 verschiedenen Arten bestand und im August des Vorjahrs (2014) ausgesät wurde; zuvor war Winterweizen geerntet worden. Die Düngung bestand aus $160 \mathrm{~kg} / \mathrm{ha} \mathrm{N}$ (Alzon; ammoniumhaltiger stabilisierter Stickstoffdünger; $46 \%$ N), $60 \mathrm{~kg} / \mathrm{ha} \mathrm{P}_{2} \mathrm{O}_{5}, 100 \mathrm{~kg} / \mathrm{ha} \mathrm{K}_{2} \mathrm{O}$ und $10 \mathrm{~kg} / \mathrm{ha} S$, die als Unterfußdüngung gegeben wurden. Die Ernte erreichte in diesem Jahr $140 \mathrm{dt} / \mathrm{ha}$. Die Wuchshöhe war mit $230 \mathrm{~cm}$ eher niedrig.

\section{Ergebnisse und Diskussion}

\subsection{Bodendaten}

Die Bodendaten der Profilgruben sind in Tabelle 1 dargestellt. Der pH-Wert an den einzelnen Standorten war neutral bis schwach sauer, von $\mathrm{pH}$ 6,5 in den oberen bis $\mathrm{pH}$ 5,6 in den tieferen Bodenschichten. Die Werte liegen im günstigen $\mathrm{pH}$-Bereich für landwirtschaftliche Kulturen (BMNT, 2015). Ein Einfluss der unterschiedlichen Bodenbewirtschaftungsvarianten war nicht ersichtlich. Allerdings waren die beiden untersuchten Hopfengärten durch einen Weg voneinander getrennt. Dieser Weg, welcher laut Aussagen des bearbeitenden Landwirts schon lange besteht, hat eine Art künstliche Hangsohle (Kolluvium) geschaffen.

Die elektrische Leitfähigkeit (EL) ist ein Maß für den Salzgehalt bzw. die Versalzung des Bodens und ist ein Indikator für die Wachstumsbedingungen. Hoher Salzgehalt reduziert die Verfügbarkeit des Wassers für die Pflanzen und induziert physiologische Trockenheit. Wenn große Mengen an Salz in den Transpirationsstrom gelangen, werden auch die Pflanzenzellen verletzt. Die Symptome sind eingeschränktes Wurzelwachstum, gebräunte Blattspitzen bis zu reduzierten Erträgen. Böden werden als versalzt bezeichnet, wenn die EL der Bodenlösung bei Wassersättigung $4 \mathrm{dS} / \mathrm{m}(=\mathrm{mS} / \mathrm{cm})$ überschreitet (Richards, 1954). Die salzempfindlichen Kulturpflanzen reagieren jedoch schon bei einer EL von 1,5 dS/m mit Ertragsabnahme (Tanji und Kielen, 2002). Aufgrund der Ergebnisse, die weit unter den obengenannten Grenzwerten lagen, war keine negative Auswirkung der Salzkonzentrationen auf

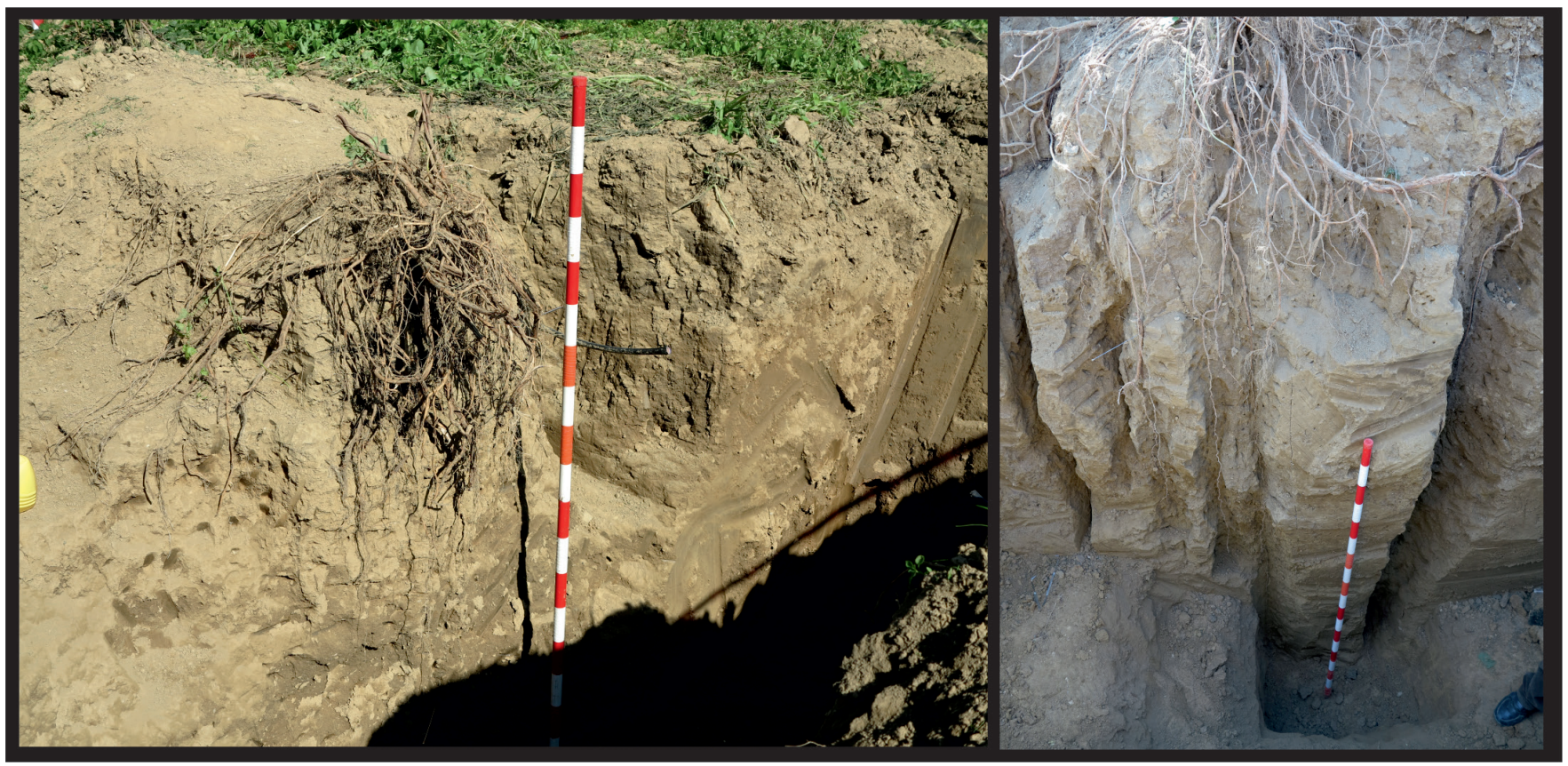

Abbildung 2. Wurzelaufnahmen von Hopfen: Oberirdisch schwach entwickelte bewässerte Pflanze mit einer Wurzeltiefe von $130 \mathrm{~cm}$ und einer seitlichen Ausbreitung von $160 \mathrm{~cm}$ (links) sowie oberirdisch gut entwickelte nicht bewässerte Pflanze mit einer Wurzeltiefe von $370 \mathrm{~cm}$ und einer seitlichen Ausbreitung von $350 \mathrm{~cm}$ (rechts).

Figure 2. Root picture of hop: above ground poorly developed irrigated plant with rooting depth of $130 \mathrm{~cm}$ and side spread of $160 \mathrm{~cm}$ (left) and above ground well developed non-irrigated plant with rooting depth of $370 \mathrm{~cm}$ and side spread of $350 \mathrm{~cm}$ (right). 
Tabelle 1. Bodendaten des Hopfen- und Maisstandortes Wolnzach

Table 1. Soil data for hop and maize location Wolnzach

\begin{tabular}{|c|c|c|c|c|c|c|c|c|c|c|c|}
\hline Tiefe & $\begin{array}{l}\text { Wasser- } \\
\text { gehalt }\end{array}$ & $\begin{array}{l}\text { pH- } \\
\text { Wert }\end{array}$ & $\begin{array}{l}\text { Elektrische } \\
\text { Leitfähigkeit }\end{array}$ & $\mathbf{N}_{\text {ges }}$ & $\mathrm{C}_{\mathrm{ges}}$ & $\mathrm{C}_{\text {org }}$ & $\mathrm{C}_{\text {anorg }}$ & $\mathbf{P}_{\text {ges }}$ & Sand & Schluff & Ton \\
\hline $\mathrm{cm}$ & $\%$ & $\mathrm{CaCl}_{2}$ & $\mathrm{mS} / \mathrm{cm}$ & $\%$ & $\%$ & $\%$ & $\%$ & $\begin{array}{c}m g P_{2} \mathrm{O}_{5} / \\
1000 g \\
\text { Boden }\end{array}$ & $\%$ & $\%$ & $\%$ \\
\hline \multicolumn{12}{|c|}{ Hopfen unbewässert } \\
\hline $0-30$ & 13,4 & 6,11 & 0,257 & 0,19 & 1,90 & 1,49 & 0,42 & 117 & 24,8 & 61,0 & 14,2 \\
\hline $30-50$ & 13,5 & 6,13 & 0,137 & 0,06 & 0,45 & 0,02 & 0,43 & 107 & 10,6 & 71,1 & 18,3 \\
\hline $110-130$ & 12,0 & 6,08 & 0,095 & 0,07 & 0,40 & 0,05 & 0,35 & 85 & 15,2 & 68,4 & 16,4 \\
\hline $130-200$ & 15,3 & 5,79 & 0,061 & 0,07 & 0,15 & 0,00 & 0,15 & 109 & 16,0 & 59,4 & 24,6 \\
\hline \multicolumn{12}{|c|}{ Hopfen bewässert } \\
\hline $0-20$ & 13,4 & 6,13 & 0,251 & 0,20 & 1,84 & 1,51 & 0,33 & 73 & 17,3 & 68,3 & 14,4 \\
\hline $20-50$ & 10,1 & 6,50 & 0,207 & 0,14 & 1,08 & 0,76 & 0,32 & 80 & 23,4 & 59,2 & 17,4 \\
\hline $100-110$ & 16,9 & 6,05 & 0,100 & 0,10 & 0,48 & 0,01 & 0,47 & 71 & 18,5 & 63,5 & 18,0 \\
\hline \multicolumn{12}{|c|}{ Mais } \\
\hline $0-30$ & 13,5 & 6,59 & 0,175 & 0,16 & 1,19 & 0,77 & 0,42 & 93 & 57,2 & 30,1 & 12,7 \\
\hline $30-60$ & 10,8 & 5,98 & 0,105 & 0,11 & 0,47 & 0,22 & 0,25 & 82 & 40,9 & 43,5 & 15,6 \\
\hline $60-100$ & 10,5 & 5,76 & 0,091 & 0,09 & 0,39 & 0,14 & 0,25 & 80 & 48,1 & 38,8 & 13,1 \\
\hline $100-140$ & 14,8 & 5,60 & 0,092 & 0,10 & 0,37 & 0,06 & 0,30 & 89 & 48,8 & 40,2 & 11,0 \\
\hline
\end{tabular}

die Wurzel- und Pflanzenentwicklung abzuleiten. Es wurde ebenfalls kein wesentlicher Unterschied zwischen den beiden Hopfenstandorten festgestellt.

Der gemessene Gesamtstickstoffgehalt $\left(\mathrm{N}_{\text {ges }}\right)$ des Bodens umfasst den organisch gebundenen Stickstoff und anorganische Formen, vor allem Ammonium und Nitrat. Auf allen Flächen nahm der Nges mit zunehmender Bodentiefe von $0,20 \%$ auf bis zu 0,07 \% ab. Der Gesamtkoh- lenstoff des Bodens $\left(\mathrm{C}_{\text {ges }}\right)$ ist in den verschiedenen organischen und anorganischen Formen (z. B. Carbonaten) enthalten. Für den oberen Boden $(0-30 \mathrm{~cm})$ war der Anteil des organischen Kohlenstoffes $\left(\mathrm{C}_{\text {org }}\right)$ am höchsten und allgemein zu niedrig für eine landwirtschaftlich genutzte Fläche. Der $\mathrm{C}_{\text {org }}$ definiert die Bodenfruchtbarkeit und ist von essenzieller Bedeutung für die Struktur und das Wasserspeichervermögen des Bodens (Hudson, 1994; Six et

Tabelle 2. Mais: Seitenwurzel einer Sprosswurzel des obersten Kranzes

Table 2. Maize: lateral root axis of a young shoot root from the top crown

\begin{tabular}{|c|c|c|c|c|c|}
\hline \multicolumn{6}{|l|}{ Seitenwurzel 1. Ordnung } \\
\hline Länge d. Seitenwurzel & $\begin{array}{l}\text { Gesamtlänge } \\
\text { inkl. Verzweig. }\end{array}$ & $\begin{array}{c}\text { Oberfläche } \\
\text { d. Seitenwurzel }\end{array}$ & $\begin{array}{l}\text { Gesamtoberfläche } \\
\text { inkl. Verzweig. }\end{array}$ & Mittlerer Durchmesser & $\begin{array}{l}\text { Mittlerer Durchmesser } \\
\text { inkl. Verzweig. }\end{array}$ \\
\hline $\mathrm{cm}$ & $\mathrm{cm}$ & $\mathrm{cm}^{2}$ & $\mathrm{~cm}^{2}$ & $m m$ & $m m$ \\
\hline 110,6 & 554,3 & 59,9 & 132,7 & 1,7 & 0,8 \\
\hline \multicolumn{6}{|c|}{ Verzweigungen: Wurzeln 2.+3. Ordnung } \\
\hline Länge einzelner Wurzeln & Gesamtlänge & & Gesamtoberfläche & $\begin{array}{c}\text { Mittlerer } \\
\text { Durchmesser }\end{array}$ & \\
\hline $\mathrm{cm}$ & $\mathrm{cm}$ & & $\mathrm{cm}^{2}$ & $m m$ & \\
\hline $0,3-5,4$ & 361,1 & & 57,4 & 0,5 & \\
\hline \multicolumn{6}{|c|}{ Verzweigungen: Wurzeln 4. Ordnung } \\
\hline & Gesamtlänge & & Gesamtoberfläche & Mittlerer Durchmesser & \\
\hline & $\mathrm{cm}$ & & $\mathrm{cm}^{2}$ & $m m$ & \\
\hline & 82,7 & & 4,1 & 0,2 & \\
\hline
\end{tabular}


al., 2000). Der gemessene Phosphorgehalt lag zwischen 80 und $120 \mathrm{mg} / 1000 \mathrm{~g}$ Boden und war auf allen Flächen im oberen Bereich der ausreichenden Versorgungsstufe C (BMLFUW, 2017).

Die Bodentextur des Hopfenstandortes zeigt eine Dominanz der Schuff-Fraktion, was auf das Ausgangsmaterial Löss/Lösslehm hinweist. Typisch für die Parabraunerde ist die etwas geringere Tonfraktion im Oberboden, verglichen mit dem darunterliegenden Bt-Horizont. Der Maisstandort befand sich auf einem deutlich leichteren Boden mit hohem Sandanteil und einem mittleren Tongehalt von nur $13,1 \%$.

\subsection{Hopfenwurzeln}

Die beiden Hopfenstandorte wiesen eine sehr unterschiedliche Wurzelausbreitung auf (Abbildungen 2 und 3). Die Wuchshöhe betrug $7 \mathrm{~m}$ und war annähernd gleich. Die Bewurzelung des Hopfens ist sprossbürtig, da die Jungpflanzen vegetativ vermehrt werden und somit die ersten Wurzeln aus dem Spross entspringen. Die ersten 2-3 Wurzeln wachsen zuerst unverzweigt pfahlartig nach unten. Erst nach einigen Wochen bilden sich weitere sprossbürtige Wurzeln.

Die Wurzelausbreitung in drei Zonen, wie sie von Graf et al. (2014) beschrieben wurde, ist bei der unbewässerten Pflanze deutlicher zu sehen als bei der bewässerten (Abbildungen 3a und 3b). Die Wurzeltiefe der bewässerten Variante reichte nur bis $130 \mathrm{~cm}$, während die Wurzeln der unbewässerten Pflanze bis in eine Tiefe von $370 \mathrm{~cm}$ zu finden waren. Die seitliche Ausbreitung lag vorwiegend in einer Bodentiefe zwischen 20 und $40 \mathrm{~cm}$. Ein Großteil dieser Wurzeln war mehrjährig. Der Durchmesser der meisten dieser Wurzeln lag zwischen 1,2 bis $20 \mathrm{~mm}$. Nur einige Wurzeln nahe der Basis erreichten eine Dicke von bis zu $35 \mathrm{~mm}$. Weiße, junge Wurzeln mit Durchmessern von 0,2 bis $1,2 \mathrm{~mm}$ waren im gesamten durchwurzelten Raum zu finden und entsprangen jeweils aus den mehrjährigen bereits lignifizierten Wurzeln. Der Verzweigungsgrad reicht bis zur 5. Ordnung, mitunter sogar bis zur 6. Ordnung. Die Wachstumsrichtungen der tief und seitwärts wachsenden Wurzeln reichten von senkrecht abwärts, eben über schräg abwärts oder mitunter auch bis zu senkrecht aufwärts. Nur in der obersten bearbeiteten Zone in den Hopfenreihen (Bifang) konnte für die jungen dünnen Wurzeln, die sich am unteren Teil der hochwachsenden Sprosse entwickeln, kein Unterschied für die beiden Varianten gefunden werden.
Auf der bewässerten Fläche fiel auf, dass nicht die dickeren Wurzeln, sondern eher die dünneren, noch hellen bis weißen Wurzeln die größten Tiefen erreichten. Bei mehreren Wurzeln ließen sich deutliche Absterbeerscheinungen beobachten. Die intensivste Bewurzelung lag in einer Bodentiefe zwischen 0 und $40 \mathrm{~cm}$. Die größten Wurzeldicken lagen bei $23 \mathrm{~mm}$. An mehreren Stellen ließen sich deutliche Verdickungen und Verdünnungen beobachten (Abbildung 4).

Die anatomischen Untersuchungen zeigten, dass die Breite des Holzteiles und die Weite der Xylem-Leitelemente der Wurzeln aus der bewässerten als auch der unbewässerten Fläche, trotz der Veränderung der Wurzeldicke, weitgehend gleich blieben. Die Zunahme der Dicke betraf vor allem die
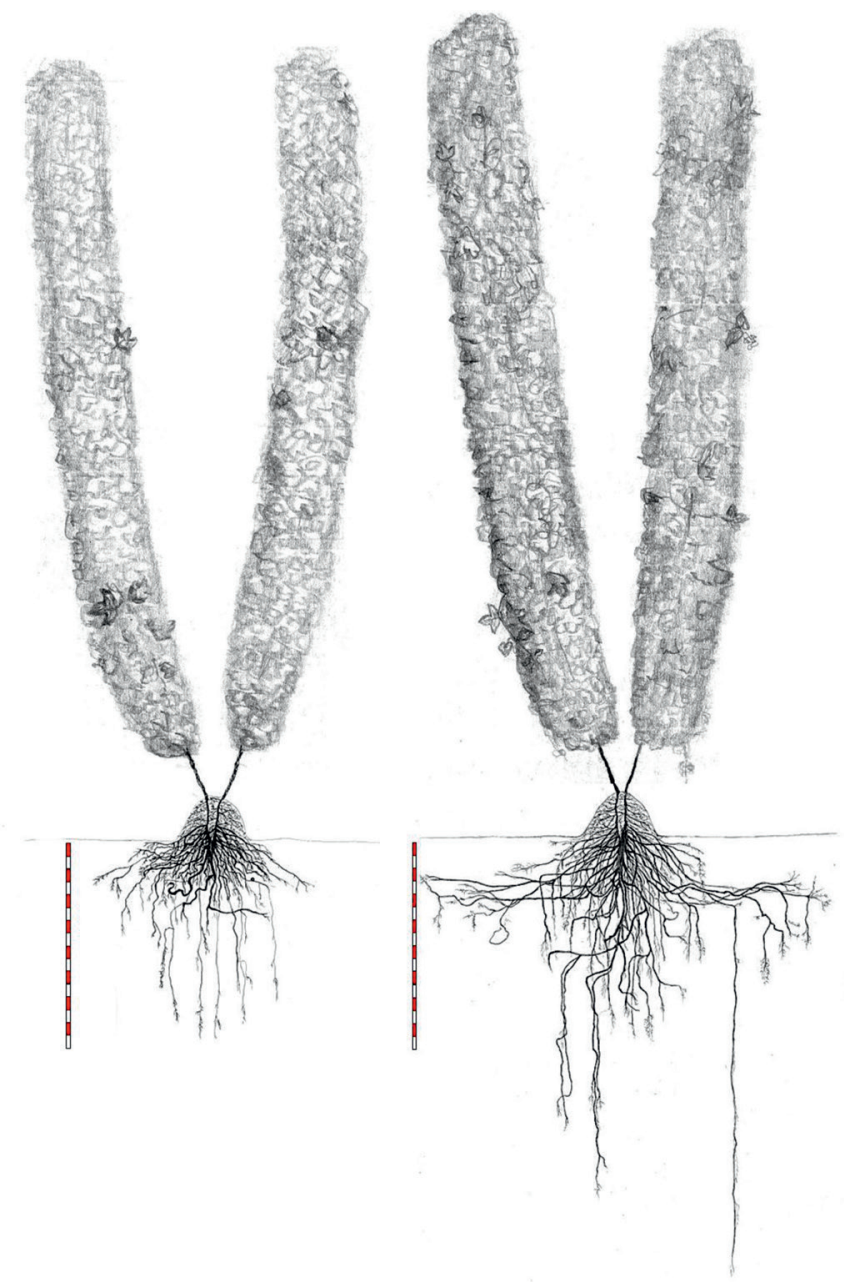

Abbildung 3. Feldaufnahme der Hopfenwurzel (Handzeichnung): a) bewässert und b) ohne Bewässerung

Figure 3. Hand drawing of hop roots: a) irrigated and b) non-irrigated 


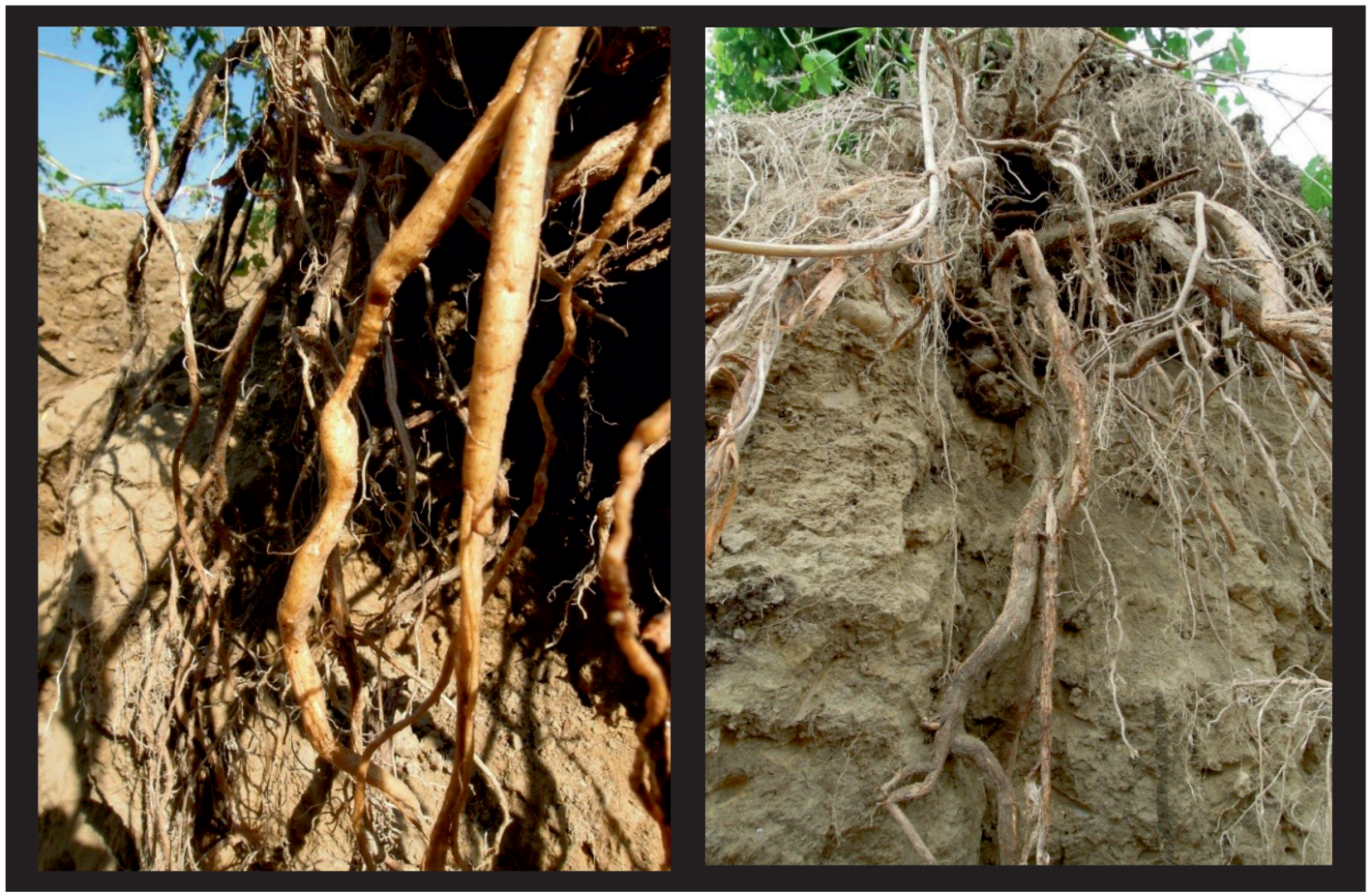

Abbildung 4. Bewässerte Hopfenpflanze mit Verdünnungen und Verdickungen (links), im unbewässerten Standort (rechts) sind diese kaum sichtbar. Figure 4. Irrigated hop plant with thinnings and thicknings (left), for non-irrigated (right) site this is hardly visible.

Zunahme an Bastteil, der zum Teil auf die Vergrößerung der Grundgewebszellen im Bastteil zurückzuführen ist.

Alle untersuchten Schnitte zeigten eine diarche Leitstrangverteilung. Zwischen den beiden Xylempolen war überall ein relativ großes Xylem-Leitelement zu sehen (Abbildung 5, links). Miller (1958) fand diese auch fallweise triarch. Auch bei den Wurzeln, die bereits die primäre Rinde verloren und bereits einen erheblichen sekundären Zuwachs zeigten, waren die diarchen Leitstränge noch deutlich zu erkennen (Abbildung 5, rechts). Für die große Wuchsfreudigkeit der Hopfenpflanzen spricht auch der deutlich höhere Anteil an Bast, das breite Kambium zwischen Xylem und Phloem, die hohe Reservestoffspeicherung von Stärke und das Vorkommen von Ölzellen (Vriet et al., 2014).

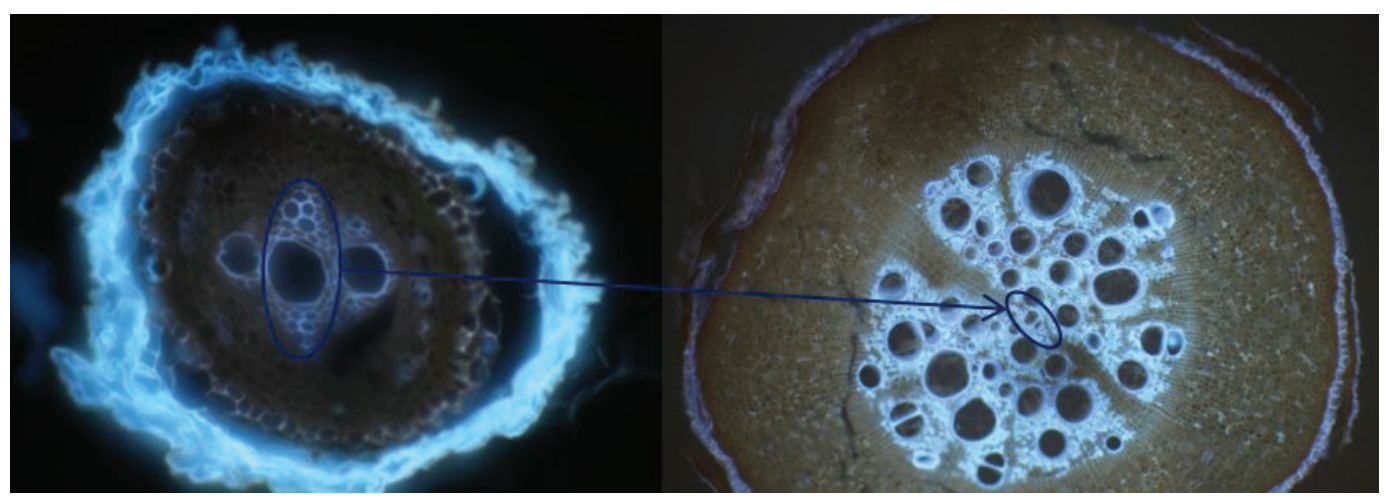

Abbildung 5. Anatomische Querschnitte von Hopfenwurzeln: links) Jugendstadium aus $370 \mathrm{~cm}$ Tiefe, $\mathrm{d}=0,55 \mathrm{~mm}, \mathrm{max} . \mathrm{Gefäß} \mathrm{d}=80 \mu \mathrm{m}$, die primäre Rinde der Probe war stark geschrumpft; rechts) Fortgeschrittenes Alter aus $230 \mathrm{~cm}$ Tiefe von der gleichen Wurzel, $\mathrm{d}=2 \mathrm{~mm}$, max. Gefäß d = $294 \mu \mathrm{m}$

Figure 5. Anatomical cross section of hop root: left) juvenile stage from $370 \mathrm{~cm}$ depth, $\mathrm{d}=0.55 \mathrm{~mm}$, max. vessel $\mathrm{d}=80 \mu \mathrm{m}$, the primary bark was severely shrunk; right) advanced age from $230 \mathrm{~cm}$ depth from the same axis, $\mathrm{d}=2 \mathrm{~mm}$, max. vessel $\mathrm{d}=294 \mu \mathrm{m}$ 


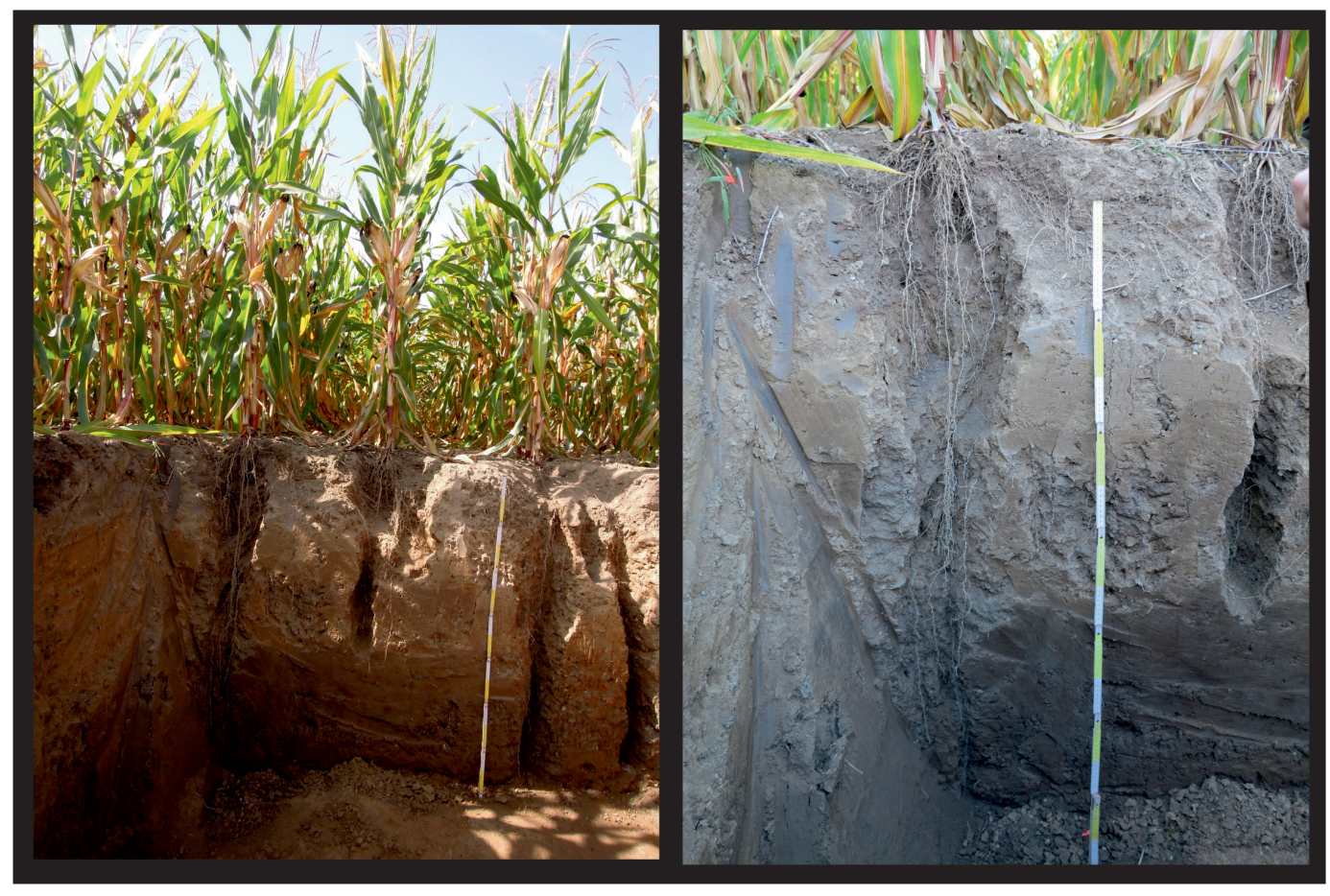

Abbildung 6. Wurzelaufnahmen von Mais: Profil (links) und im Detail (rechts)

Figure 6. Root picture of maize: soil profile (left) and a detail (right)

\subsection{Maiswurzeln}

Bei der Maisausgrabung fiel besonders das Tiefenwachstum zahlreicher Seitenwurzeln 1. Ordnung und der Sprosswurzeln im Vergleich zu früheren Ausgrabungen in Kärnten und dem Burgenland auf. Bei mehreren freigelegten Seitenwurzeln konnte ein weitgehend ebenes Wachstum bis $40 \mathrm{~cm}$ und im Anschluss daran ein senkrechter Verlauf bis in $140 \mathrm{~cm}$ Tiefe verfolgt werden (Abbildung 6).

Anhand einer Seitenwurzel 1. Ordnung (Abbildung 7) wurde die morphologische Struktur der Wurzelachse genauer beschrieben (Tabelle 2). Der mittlere Durchmesser der Achsen nimmt zwischen den Ordnungen (1. vs. $2 .+3$. Ordnung; $2 .+3$. Ordnung vs. 4. Ordnung) um jeweils ca. $70 \%$ ab. Die Verzweigungen 2. und 3. Ordnung machen den Großteil der Länge der Wurzelachsen aus (65\%), während der Hauptstrang 1. Ordnung $20 \%$ und die Verzweigungen 4. Ordnung $15 \%$ der Gesamtlänge bilden. Die Wurzelachsen 1.-3. Ordnung bilden mit etwa gleichem Anteil den Großteil der Wurzeloberfläche aus, während die feinen und kurzen Achsen höchster Ordnung nur geringfügig zur Gesamtoberfläche beitragen.

\section{Zusammenfassung}

Am Hopfenstandort war das deutlich schwächere oberirdische Wachstum der bewässerten Variante im Vergleich zu üppig entwickelten nicht bewässerten Pflanzen in einer nur geringen Entfernung zu beobachten. Aus den Ergebnissen der Wurzelausgrabungen war deutlich zu erkennen, dass sich die größere Ausbreitung der nicht bewässerten Hopfenpflanze nach der Tiefe günstig auf das oberirdische Wachstum auswirkte. Für die deutlich schwächere Wurzelausbreitung nach der Tiefe und den Seiten spielen die häufig beobachteten Absterbeprozesse von den Wurzelenden her eine wesentliche Rolle. Die Ursachen dafür müssen allerdings noch ergründet werden. Als eine Ursache könnte die hohe Empfindlichkeit gegenüber Staunässe, verbunden mit schlechter Sauerstoffversorgung, sein. Auffallend war auch, dass sich die Wurzeln nicht in Richtung der Bewässerungslöcher hin entwickelten. Der nahverwandte Hanf (Cannabis sativa), der insbesondere im primären Zustand große Ähnlichkeiten zeigt, reagiert ebenfalls sehr empfindlich auf Staufeuchte und Sauerstoffarmut (Kutschera et al., 2009).

Graf et al. (2014) weisen auf das hohe durchwurzelte Bodenvolumen bei Hopfen und die entsprechende Fähigkeit 


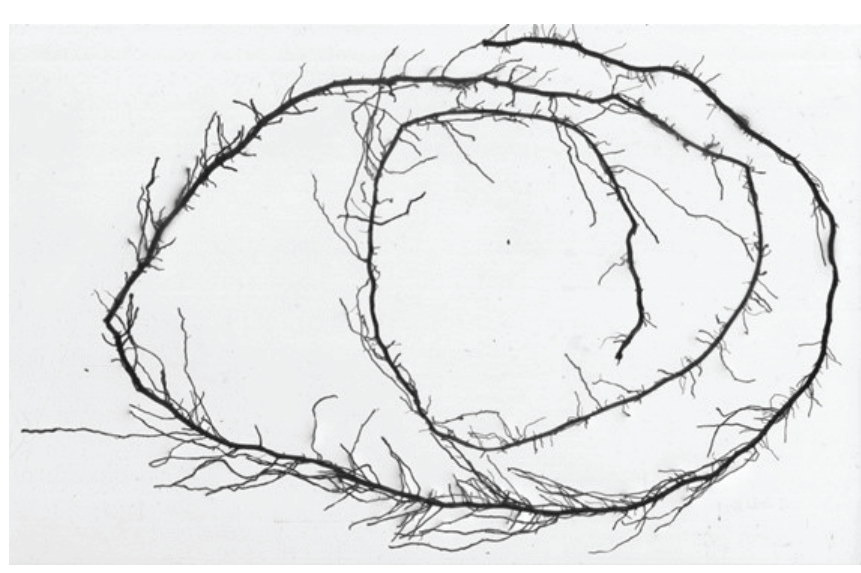

Abbildung 7. Wurzelscan (WinRhizo) einer Wurzelachse 1. Ordnung von Mais

Figure 7. Root scan (WinRhizo) of a first order root axis of maize

der Erschließung der Bodenwasservorräte hin. Die Bewässerung einer Kultur mit hohem Durchwurzelungspotenzial in einem Gebiet mit mittleren Gesamtniederschlägen von $780 \mathrm{~mm}$ und gut speicherfähigen Böden scheint damit eine nur sehr bedingt sinnvolle Managementmaßnahme. Die vorliegenden Untersuchungen weisen sogar auf das Risiko eines geringeren Wurzeltiefgangs sowie temporären Luftmangels in der Wurzelzone hin, die den erwarteten positiven Effekt einer Zusatzbewässerung aufheben und sogar zu geringerer Vitalität und niedrigeren Erträgen bewässerter Hopfenanlagen führen können.

Der Mais war bei relativ geringer Wuchshöhe auffällig gleichmäßig über die Tiefe durchwurzelt. Während die Rolle der Interaktion zwischen Bodenwasserverfügbarkeit und Wurzelverteilung auf die Funktionalität des Wurzelsystems bereits häufig gezeigt wurde (z. B. Leitner et al., 2014), sollte im gemäßigten Klimagebiet, insbesondere auf temporär zur Staunässe neigenden Böden, die Regelung des Bodenlufthaushaltes für die physiologische Funktionalität der Wurzeln stärkere Beachtung finden.

Zusammenfassend kann gefolgert werden, dass auch eine partielle Wurzelfreilegung einige Rückschlüsse von Umwelteinflüssen auf das Pflanzenwachstum erlaubt.

\section{Danksagung}

An dieser Stelle möchten wir uns bei den beiden Landwirten Herrn Florian Bogensberger und Herrn Georg Thalmaier für die Bereitstellung der Flächen für die Wurzelausgrabungen sowie bei Herrn Max Stadler für die orga- nisatorische Vorbereitung und die tatkräftige Mithilfe sehr herzlich bedanken.

\section{Literaturverzeichnis}

Bayerische Landesanstalt für Landwirtschaft (2015): Hopfen 2015 - Anbau, Sorten, Düngung, Pflanzenschutz, Ernte. Grünes Heft 2015, Institut für Pflanzenbau und Pflanzenzüchtung, Arbeitsbereich Hopfen, Bayerische Landesanstalt für Landwirtschaft, Wolnach, Deutschland, $126 \mathrm{~S}$.

BMNT (2015): Düngung im Hopfenbau. Bundesministerium für Nachhaltigkeit und Tourismus, Österreich. https://www.bmnt.gv.at/land/produktion-maerkte/ pflanzliche-produktion/boden-duengung/Bodenschutz. html. Abgerufen am 16. Jänner 2018.

BMLFUW (2017): Richtlinien für die sachgerechte Düngung im Ackerbau und Grünland. Anleitung zur Interpretation von Bodenuntersuchungsergebnissen in der Landwirtschaft. 7. Aufl., Bundesministeriums für Land- und Forstwirtschaft, Umwelt und Wasserwirtschaft, $115 \mathrm{~S}$.

Bodner, G., Nakhforoosh, A. und H.-P. Kaul (2015): Management of crop water under drought: a review. Agronomy for Sustainable Development 35, 401-442.

Braune, W., Lemann, A. und H. Taubert (1999): Pflanzenanatomisches Praktikum I: die Anatomie der Vegetationsorgane der Samenpflanzen. 8. Aufl., G. Fischer, Jena, Deutschland, 368 S.

CLIMATE-DATA.ORG (2015): http://de.climate-data. org/location/12867/. Abgerufen am 16. Dezember 2015.

Graf, T., Beck, M., Mauermeier, M., Ismann, D., Portner, J., Doleschel, P. und U. Schmidhalter (2014): Humulus lupulus - The Hidden Half. BrewingScience 67, 161-167.

Himmelbauer M.L., Sobotik, M. und W. Loiskandl (2012): No-tillage farming, soil fertility and maize root growth. Archives of Agronomy and Soil Science 58 (SUPPL), 151-157.

Hudson, B.D. (1994): Soil organic matter and available water capacity. Journal of Soil and Water Conservation 49, 189-194.

Kohlmann, H. und A. Kastner (1975): Der Hopfen. Hopfen-Verlag, Wolnzach, Deutschland, $43 \mathrm{~S}$.

Kutschera, L., Lichtenegger, E. und M. Sobotik (2009):

Wurzelatlas der Kulturpflanzen gemäßigter Gebiete 
mit Arten des Feldgemüsebaues. DLG, Frankfurt/M., Deutschland, 527 S.

Leitner, D., Meunier, F., Bodner, G., Javaux, M. und A. Schnepf (2014): Impact of contrasted maize root traits at flowering on water stress tolerance - A simulation study. Field Crops Research, 165, 125-137.

Lynch, J. (1995): Root architecture and plant productivity. Plant Physiology 109, 7-13.

Miller, R.-H. (1958): Morphology of Humulus lupulus. I. Developmental anatomy of the primary root. American Journal of Botany 45, 418-431.

Richards, L.A. (Ed.) (1954): Diagnosis and improvement of saline and alkali soils. USDA Agricultural Handbook 60, U. S. Salinity Laboratory, Riverside, CA, USA.
Rid, H. (1966): Qualität durch standortgerechten Hopfenanbau. Hopfenrundschau 13, 224-229.

Six, J., Paustian, K., Elliott, E.T. und C. Combrink (2000): Soil structure and organic matter I. Distribution of aggregate-size classes and aggregate-associated carbon. Soil Science Society of America Journal 64, 681-689.

Tanji, K.K. und N.C. Kielen (2002): Agricultural drainage water management in arid and semi-arid areas. FAO Irrigation and Drainage Paper 61, 205 S.

Vriet, C., Smith, A.M. und T.L. Wang (2014): Root starch reserves are necessary for vigorous re-growth following cutting back in Lotus japonicus. PLoS One 9, e87333. 